\title{
Top quark as resonance: renormalization and spin effect
}

\author{
A.E. Kaloshin ${ }^{a}$ and V.P. Lomov ${ }^{* b c}$ \\ ${ }^{a}$ Irkutsk State University, Irkutsk \\ ${ }^{b}$ Institute for System Dynamics and Control Theory of SB RAS, Irkutsk \\ ${ }^{c}$ Irkutsk State Technical University, Irkutsk \\ E-mail: shin7@rambler.ru, V.P.Lomovegmail.com
}

We suggest the description of the dressed fermion propagator with parity non-conservation in the form with separated positive and negative energy poles. We found general form of the $\gamma$-matrix off-shell projectors and corresponding resonance factors. The parity violation leads to deviation of resonance factors from the naive Breit-Wigner form and to appearance of non-trivial spin corrections. However, for top quark with SM vertex the resonance factor returns to the standard one due to $\Gamma / m \ll 1$.

XXI International Baldin Seminar on High Energy Physics Problems, September 10-15, 2012

JINR, Dubna, Russia

\footnotetext{
* Speaker.
} 


\section{Introduction}

The top quark, the heaviest elementary particle observed to date, plays a special role in Standard Model (SM) [1-3] and it is an object of intensive research at LHC [4-8]. Being a shortliving particle (due to the open channels with W-boson on mass shell), it may be considered on an equal footing with ordinary hadron resonances. The dressed propagator can be obtained as a result of Dyson summation of self-energy insertions or, equivalently, by solving the Dyson-Schwinger equation. As for top quark, its vertex violates parity, so $\gamma^{5}$ takes part in this process, and it leads to nonstandard form of resonance factor, as we shall see below.

The form of fermion resonance with parity violation was discussed earlier. In particular, in [9] were written general formulas for dressed propagator with the use of the off-shell basis. The paper [10] was devoted to extension of the concept of pole mass and width [11-15] to the case of the parity violation. The obtained dressed fermion propagator was written in a boson-like form without separation of the positive and negative energy poles. It is difficult to compare this general expression with the standard Breit-Wigner form, in particular to recognize there the on-shell decay width.

In this work we build the $\gamma$-matrix projectors onto the positive and negative energy poles and corresponding resonance factors. The key moment is the use of the spectral representation of operator for this purpose. The explicit form of this representation (4.5), (4.6) can be obtained for arbitrary form of interaction, its particular case (5.7) corresponds to V-A vertex of SM.

\section{Standard Breit-Wigner formula in QFT}

To obtain Breit-Wigner-like formula in QFT one needs to solve the Dyson-Schwinger equation for the dressed propagator,

$$
G=G_{0}+G_{0} \Sigma G, \quad \text { or } \quad G^{-1}=G_{0}^{-1}-\Sigma,
$$

where $G$ and $G_{0}$ are dressed and free propagators and $\Sigma$ is a self-energy.

For bosons one has

$$
G_{0}=\frac{1}{m_{0}^{2}-s-\imath \varepsilon} \quad \text { and } \quad G=\frac{1}{m_{0}^{2}-s-\Sigma(s)} \sim \frac{1}{m^{2}-s-\imath \Gamma m},
$$

and if $\Sigma$ has imaginary part, the dressed propagator $G$ should be compared with relativistic Breit-Wigner formula for renormalization.

For fermions all are similar:

$$
G_{0}=\frac{1}{\hat{p}-m_{0}} \quad \text { and } \quad G=\frac{1}{\hat{p}-m_{0}-\Sigma(p)},
$$

but to make this procedure more transparent, it is convenient to pass to off-shell projection operators.

Let's define off-shell projection operators as follows:

$$
\Lambda^{ \pm}=\frac{1}{2}\left(1 \pm \frac{\hat{p}}{W}\right)
$$


where $W=\sqrt{p^{2}}$ is invariant mass or rest-frame energy.

In this basis dressing of fermion propagator looks like

$$
\begin{aligned}
& G_{0}=\frac{1}{\hat{p}-m_{0}}=\Lambda^{+} \frac{1}{W-m_{0}}+\Lambda^{-} \frac{1}{-W-m_{0}}, \quad \text { and } \\
& G=\Lambda^{+} \frac{1}{W-m_{0}-\Sigma_{1}(W)}+\Lambda^{-} \frac{1}{-W-m_{0}-\Sigma_{2}(W)},
\end{aligned}
$$

where the self-energy is also decomposed in this basis

$$
\Sigma(p)=A\left(p^{2}\right)+\hat{p} B\left(p^{2}\right)=\Lambda^{+}(A+W B)+\Lambda^{-}(A-W B) \equiv \Lambda^{+} \Sigma_{1}(W)+\Lambda^{-} \Sigma_{2}(W) .
$$

The positive energy pole should be compared with Breit-Wigner formula

$$
\frac{1}{W-m_{0}-\Sigma_{1}(W)} \sim \frac{1}{W-m+\imath \Gamma / 2} .
$$

The above formulas correspond to the parity conservation, because the self-energy does not involve $\gamma^{5}$.

\section{Dressed fermion propagator with parity violation}

In case of parity violation the projection basis (2.2) must be supplemented by elements with $\gamma^{5}$, it is handy to choose the basis as [9]

$$
\mathscr{P}_{1}=\Lambda^{+}, \quad \mathscr{P}_{2}=\Lambda^{-}, \quad \mathscr{P}_{3}=\Lambda^{+} \gamma^{5}, \quad \mathscr{P}_{4}=\Lambda^{+} \gamma^{5}
$$

Now the decomposition of a self-energy or a propagator has four terms

$$
S=\sum_{M=1}^{4} S_{M} \mathscr{P}_{M}
$$

where coefficients $S_{M}$ are followed by obvious symmetry properties

$$
S_{2}(W)=S_{1}(-W), \quad S_{4}(W)=S_{3}(-W) .
$$

With the use of decomposition (3.2), the Dyson-Schwinger equation (2.1) is reduced to the set of equations for scalar coefficients

$$
S_{M}=\left(S_{0}\right)_{M}-\Sigma_{M}, \quad M=1, \ldots, 4,
$$

where $\left(S_{0}\right)_{M}$ and $S_{M}$ are coefficients of free and dressed inverse propagators correspondingly. Considering the self-energy $\Sigma$ as a known value, we obtain the dressed propagator

$$
G=\sum_{M=1}^{4} G_{M} \mathscr{P}_{M}
$$

where the coefficients $G_{M}$ are

$$
G_{1}=\frac{S_{2}}{\Delta}, \quad G_{2}=\frac{S_{1}}{\Delta}, \quad G_{3}=-\frac{S_{3}}{\Delta}, \quad G_{4}=-\frac{S_{4}}{\Delta},
$$

and $\Delta=S_{1} S_{2}-S_{3} S_{4}$.

In spite of simple answer (3.4), it is inconvenient because the positive and negative energy poles are not separated, compare with formula (2.3). So, in case of the parity non-conservation the comparison with Breit-Wigner formula is not so evident. 


\section{Spectral Representation of propagator}

In order to obtain the analog of representation (2.3) in case of parity violation, we use the spectral representation of inverse propagator

$$
S=\lambda_{1} \Pi_{1}+\lambda_{2} \Pi_{2},
$$

where $\Pi_{k}$ are projectors, satisfying the eigenstate problem

$$
S \Pi_{k}=\lambda_{k} \Pi_{k} .
$$

Let's write the dressed propagator $S(p)$ as

$$
S=\sum_{M=1}^{4} S_{M} \mathscr{P}_{M}
$$

with arbitrary coefficients and will look for the matrix $\Pi$ in the same form with coefficients $a_{M}$.

After some algebra one finds that eigenvalues $\lambda_{i}$ are roots of the equation

$$
\lambda^{2}-\lambda\left(S_{1}+S_{2}\right)+\left(S_{1} S_{2}-S_{3} S_{4}\right)=0,
$$

and solution of (4.2) is

$$
\Pi_{i}=\mathscr{P}_{1} a_{1}^{i}+\mathscr{P}_{2} a_{2}^{i}-\frac{S_{3}}{S_{1}-\lambda_{i}} a_{2}^{i} \mathscr{P}_{3}-\frac{S_{4}}{S_{2}-\lambda_{i}} a_{1}^{i} \mathscr{P}_{4}
$$

with arbitrary coefficients $a_{1}, a_{2}$.

In order (4.4) to be a projector, $\Pi^{2}=\Pi$, we need only one additional condition

$$
a_{2}=1-a_{1} .
$$

After it the orthogonality property $\Pi_{1} \Pi_{2}=\Pi_{2} \Pi_{1}=0$ defines $a_{1}$ coefficient

$$
a_{1}^{1}=\frac{S_{2}-\lambda_{1}}{\lambda_{2}-\lambda_{1}}, \quad a_{1}^{2}=-\frac{S_{2}-\lambda_{2}}{\lambda_{2}-\lambda_{1}} .
$$

As result we have the projectors

$$
\begin{aligned}
& \Pi_{1}=\frac{1}{\lambda_{2}-\lambda_{1}}\left(\left(S_{2}-\lambda_{1}\right) \mathscr{P}_{1}+\left(S_{1}-\lambda_{1}\right) \mathscr{P}_{2}-S_{3} \mathscr{P}_{3}-S_{4} \mathscr{P}_{4}\right), \\
& \Pi_{2}=\frac{1}{\lambda_{1}-\lambda_{2}}\left(\left(S_{2}-\lambda_{2}\right) \mathscr{P}_{1}+\left(S_{1}-\lambda_{2}\right) \mathscr{P}_{2}-S_{3} \mathscr{P}_{3}-S_{4} \mathscr{P}_{4}\right),
\end{aligned}
$$

with desired properties:

- $S \Pi_{k}=\lambda_{k} \Pi_{k}$, where an eigenvalue $\lambda_{k}$ is a root of equation (4.3),

- $\Pi_{k}^{2}=\Pi_{k}$,

- $\Pi_{1} \Pi_{2}=\Pi_{2} \Pi_{1}=0$,

- $\Pi_{1}+\Pi_{2}=1$. 
The dressed propagator $G(p)$ is obtained by reversing of equation (4.1)

$$
G=\frac{1}{\lambda_{1}} \Pi_{1}+\frac{1}{\lambda_{2}} \Pi_{2}
$$

The determinant $\Delta(W)$ of $S$ is

$$
\Delta(W)=S_{1} S_{2}-S_{3} S_{4}=\left(W-m_{0}-\Sigma_{1}\right)\left(-W-m_{0}-\Sigma_{2}\right)-\Sigma_{3} \Sigma_{4},
$$

where $\Sigma_{i}(W)$ are self-energy components in the basis (3.1). Free propagator has poles at points $W=m_{0}$ and $W=-m_{0}$, the dressed one has them at $W=m$ and $W=-m$. On the other hand, $\Delta(W)$ is equal to product of eigenvalues

$$
\Delta(W)=\lambda_{1}(W) \lambda_{2}(W)
$$

so in the spectral representation of propagator (4.6) the positive and negative energy poles contributions are separated from each other. Therefore, the matrices (4.5) are projectors onto these poles.

\section{5. t-quark propagator in Standard Model}

Consider the dressing of top quark in SM. The main one-loop contribution to self-energy arises from $\mathrm{Wb}$ intermediate state

$$
\Sigma(p)=-l g^{2}\left|V_{\mathrm{tb}}\right|^{2} \int \frac{\mathrm{d}^{4} k}{(2 \pi)^{4}} \gamma^{\mu}\left(1-\gamma^{5}\right) \frac{\hat{p}-\hat{k}+m_{\mathrm{b}}}{(p-k)^{2}-m_{\mathrm{b}}^{2}} \gamma^{v}\left(1-\gamma^{5}\right) \frac{g_{\mu \nu}-k_{\mu} k_{v} / m_{\mathrm{W}}^{2}}{k^{2}-m_{\mathrm{W}}^{2}},
$$

and generates only kinetic term

$$
\Sigma(p)=\hat{p}\left(1-\gamma^{5}\right) \Sigma_{0}\left(W^{2}\right)
$$

Its decomposition in the basis (3.1) has the following coefficients:

$$
\Sigma_{1}=W \Sigma_{0}\left(W^{2}\right), \quad \Sigma_{2}=-W \Sigma_{0}, \quad \Sigma_{3}=-W \Sigma_{0}, \quad \Sigma_{4}=W \Sigma_{0} .
$$

As a preliminary, let us forget about renormalization of self-energy and calculate the eigenvalues

$$
\lambda_{1,2}=-m \pm W \sqrt{1-2 \Sigma_{0}\left(W^{2}\right)}
$$

In analogy with on-mass-shell (OMS) renormalization scheme let's subtract the real part of selfenergy at resonance point

$$
\lambda_{1,2}=-m \pm W \sqrt{1-2\left(\Sigma_{0}\left(W^{2}\right)-\operatorname{Re} \Sigma_{0}\left(m^{2}\right)\right)} .
$$

As a result we have rather unusual resonance factor

$$
\frac{1}{\lambda_{1}(W)}=\frac{1}{W \sqrt{1+l \frac{\Gamma}{m}-m}}
$$


which only at $\Gamma / m \ll 1$ returns to standard Breit-Wigner form,

$$
\frac{1}{\lambda_{1}(W)} \simeq \frac{1}{W-m+\imath W \frac{\Gamma}{2 m}} \quad \text { at } \Gamma / m \ll 1 .
$$

To analyse the obtained dressed propagator in more detail, we need to renormalize it. We will use the OMS scheme of renormalization in order to compare with Breit-Wigner formula. Let's suppose that self-energy does not have imaginary part. Then the analogy of this case with the mixing problem allows us to formulate the OMS requirements (see [16, 17]) on the self-energy

- $\Sigma_{1}$ has zero of second order at $W=m$

- $\Sigma_{3}$ has zeroes at $W=m$ and $W=-m$.

The $\Sigma_{2}$ and $\Sigma_{4}$ are defined by substitution $W \rightarrow-W$, so the OMS renormalization in this case is

$$
\begin{aligned}
& \Sigma_{1}^{\mathrm{r}}(W)=\Sigma_{1}(W)-\Sigma_{1}(m)-\Sigma_{1}^{\prime}(m)(W-m), \\
& \Sigma_{2}^{\mathrm{r}}(W)=\Sigma_{1}^{\mathrm{r}}(-W), \\
& \Sigma_{3}^{\mathrm{r}}(W)=-W\left(\Sigma_{0}\left(W^{2}\right)-\Sigma_{0}\left(m^{2}\right)\right), \\
& \Sigma_{4}^{\mathrm{r}}(W)=\Sigma_{3}^{\mathrm{r}}(-W) .
\end{aligned}
$$

Eigenvalues in OMS scheme are

$$
\lambda_{1,2}(W)=-m K \pm W K \sqrt{d}, \quad \text { where } \quad d=1-2 \widetilde{\Sigma} / K
$$

and $K=1+2 m^{2} \Sigma_{0}^{\prime}\left(m^{2}\right), \widetilde{\Sigma}=\Sigma_{0}\left(W^{2}\right)-\Sigma_{0}\left(m^{2}\right)$.

Let us write down the eigenvalues in vicinity of $W=m$

$$
\begin{aligned}
& \lambda_{1}(W)=W-m+o(W-m), \\
& \lambda_{2}(W)=-2 m K-(W-m)+o(W-m),
\end{aligned}
$$

and in vicinity of $W=-m$

$$
\begin{aligned}
& \lambda_{1}(W)=-2 m K-(-W-m)+o(-W-m), \\
& \lambda_{2}(W)=-W-m+o(-W-m) .
\end{aligned}
$$

Projectors on eigenstates have the form

$$
\begin{aligned}
& \Pi_{1}=\mathscr{P}_{1} \frac{\sqrt{d}+(1-\widetilde{\Sigma} / K)}{2 \sqrt{d}}+\mathscr{P}_{2} \frac{\sqrt{d}-(1-\widetilde{\Sigma} / K)}{2 \sqrt{d}}-\mathscr{P}_{3} \frac{\widetilde{\Sigma} / K}{2 \sqrt{d}}+\mathscr{P}_{4} \frac{\widetilde{\Sigma} / K}{2 \sqrt{d}}, \\
& \Pi_{2}=\mathscr{P}_{1} \frac{\sqrt{d}-(1-\widetilde{\Sigma} / K)}{2 \sqrt{d}}+\mathscr{P}_{2} \frac{\sqrt{d}+(1-\widetilde{\Sigma} / K)}{2 \sqrt{d}}+\mathscr{P}_{3} \frac{\widetilde{\Sigma} / K}{2 \sqrt{d}}-\mathscr{P}_{4} \frac{\widetilde{\Sigma} / K}{2 \sqrt{d}}
\end{aligned}
$$

and dressed propagator is

$$
G(p)=\frac{m_{0}+\hat{p}-\hat{p}\left(1+\gamma^{5}\right) \widetilde{\Sigma} / K}{K\left(W^{2} d-m_{0}^{2}\right)}
$$


The expressions for eigenvalues and projectors may be simplified in vicinity of $W^{2}=m^{2}$, where $\widetilde{\Sigma}(W) \ll 1$ and we take into account only linear in $\widetilde{\Sigma}$ terms

$$
\begin{aligned}
\lambda_{1,2}(W) & =K(-m \pm W) \mp W \widetilde{\Sigma}\left(W^{2}\right), \\
\Pi_{1} & =\mathscr{P}_{1}-\mathscr{P}_{3} \frac{\widetilde{\Sigma}}{2 K}+\mathscr{P}_{4} \frac{\widetilde{\Sigma}}{2 K}=\Lambda^{+}-\frac{\widetilde{\Sigma}\left(W^{2}\right)}{2 K} \frac{\hat{p} \gamma^{5}}{W}, \\
\Pi_{2} & =\mathscr{P}_{2}+\mathscr{P}_{3} \frac{\widetilde{\Sigma}}{2 K}-\mathscr{P}_{4} \frac{\widetilde{\Sigma}}{2 K}=\Lambda^{-}+\frac{\widetilde{\Sigma}\left(W^{2}\right)}{2 K} \frac{\hat{p} \gamma^{5}}{W} .
\end{aligned}
$$

Let's consider the case when the self-energy $\Sigma(W)$ acquire the imaginary part. In this situation we use a generalization $[18,19]$ of OMS scheme for unstable particles, which consists in subtraction of real part of a loop. The formulas for eigenvalues and projectors, (5.4) and (5.5), remain the same, but in this case

$$
\widetilde{\Sigma}\left(W^{2}\right)=\Sigma_{0}\left(W^{2}\right)-\operatorname{Re} \Sigma_{0}\left(m^{2}\right), \quad \text { and } \quad K=1+2 m^{2}\left(\operatorname{Re} \Sigma_{0}\right)^{\prime}\left(m^{2}\right) .
$$

Resonance factor $1 / \lambda_{1}$ in vicinity of $W=m$ practically coincides with naive expression (5.3)

$$
\frac{1}{\lambda_{1}(W)}=\frac{1}{K(W \sqrt{1-2 \widetilde{\Sigma} / K}-m)} \approx \frac{1}{K\left(W \sqrt{1+l \frac{\Gamma(W)}{K W}}-m\right)},
$$

if to introduce the energy-dependent width $\Gamma(W)=-2 W \operatorname{Im} \Sigma_{0}\left(W^{2}\right)$.

At small $\Gamma$ resonance factor returns to standard form

$$
\frac{1}{\lambda_{1}(W)} \simeq \frac{1}{W-m+i \Gamma(W) / 2} \quad \text { at } \quad W \simeq m, \quad \Gamma / m \ll 1 .
$$

Using the same approximations in projectors, we can write down a parametrization of dressed propagator in vicinity of $W=m$ :

$$
\begin{aligned}
G=\frac{1}{W-m+\imath \Gamma(W) / 2}\left(\mathscr{P}_{1}+l \frac{\Gamma(W)}{4 K W^{2}} \hat{p} \gamma^{5}\right)+ \\
\quad+\frac{1}{-2 m K-(W-m)-\imath \Gamma(W) / 2}\left(\mathscr{P}_{2}-\imath \frac{\Gamma(W)}{4 K W^{2}} \hat{p} \gamma^{5}\right) .
\end{aligned}
$$

\section{Pole Scheme and Spectral Representation}

The pole renormalization scheme for fermion with parity non-conservation have been considered in detail in work [10]. We will consider the pole scheme on the base of spectral representation. Instead of renormalization of determinant, as in [10], in this case it is sufficient to renormalize the single pole contribution $1 / \lambda_{1}(W)$. It simplifies essentially the algebraic procedure and clarifies some aspects.

The inverse propagator has the form

$$
S(p)=\hat{p}-m_{0}-\Sigma(p)=\hat{p}-m_{0}-\left(A\left(p^{2}\right)+\hat{p} B\left(p^{2}\right)+C\left(p^{2}\right) \gamma^{5}+\hat{p} \gamma^{5} D\left(p^{2}\right)\right) .
$$

In CP-symmetric theory $C\left(p^{2}\right)=0$. 
In terms of scalar functions the eigenvalues and corresponding projectors (4.5) have the form

$$
\begin{gathered}
\lambda_{1}(W)=-m_{0}-A\left(W^{2}\right)+W R\left(W^{2}\right), \\
\lambda_{2}(W)=\lambda_{1}(-W), \\
\Pi_{1}(W)=\frac{1}{2}\left[1-\gamma^{5} \frac{C\left(W^{2}\right)}{W R\left(W^{2}\right)}+\frac{\hat{p}}{W}\left(\frac{1-B\left(W^{2}\right)}{R\left(W^{2}\right)}-\gamma^{5} \frac{D\left(W^{2}\right)}{R\left(W^{2}\right)}\right)\right], \\
\Pi_{2}=\Pi_{1}(-W),
\end{gathered}
$$

where we have introduced the notation

$$
R\left(W^{2}\right)=\sqrt{\left(1-B\left(W^{2}\right)\right)^{2}-D^{2}\left(W^{2}\right)+C^{2}\left(W^{2}\right) / W^{2}} .
$$

Let's $\lambda_{1}\left(W_{1}\right)=0$, where $W_{1}=M_{p}-\imath \Gamma_{p} / 2$ :

$$
-m_{0}-A\left(W_{1}^{2}\right)+W_{1} R\left(W_{1}^{2}\right)=0 .
$$

Real part of this equality allows to get rid of $m_{0}$ in dressed propagator

$$
\begin{gathered}
S(p)=\hat{p}-\left(\tilde{A}\left(p^{2}\right)+\hat{p} B\left(p^{2}\right)+\gamma^{5} C\left(p^{2}\right)+\hat{p} \gamma^{5} D\left(p^{2}\right)\right), \\
\tilde{A}\left(p^{2}\right)=A\left(p^{2}\right)-A\left(W_{1}^{2}\right)+\left(W_{1} R\left(W_{1}^{2}\right)\right) .
\end{gathered}
$$

The imaginary part of (6),

$$
\operatorname{Im}\left(-A\left(W_{1}^{2}\right)+W_{1} R\left(W_{1}^{2}\right)\right)=0
$$

gives relation between $\Gamma_{p}$ and self-energy at pole point. In particular, in case of parity conservation it reduces to the obvious relation

$$
\operatorname{Im}\left(W_{1}-\left(A\left(W_{1}^{2}\right)+W_{1} B\left(W_{1}^{2}\right)\right)\right)=0, \quad \text { or } \quad \frac{\Gamma_{p}}{2}=-\operatorname{Im} \Sigma_{1}\left(W_{1}^{2}\right) .
$$

Let's introduce wave function renormalization constants connecting bare and renormalized fields

$$
\Psi=Z^{1 / 2} \Psi^{\mathrm{r}}, \quad \bar{\Psi}=\bar{\Psi}^{\mathrm{r}} \bar{Z}^{1 / 2}
$$

In case of parity violation $Z^{1 / 2}, \bar{Z}^{1 / 2}$ are matrices

$$
Z^{1 / 2}=\alpha+\beta \gamma^{5}, \quad \bar{Z}^{1 / 2}=\bar{\alpha}+\bar{\beta} \gamma^{5} .
$$

Renormalized inverse propagator

$$
\begin{aligned}
S^{\mathrm{r}}(p) & =\left(\bar{\alpha}+\bar{\beta} \gamma^{5}\right)\left[\hat{p}-\left(\tilde{A}+\hat{p} B+\gamma^{5} C+\hat{p} \gamma^{5} D\right)\right]\left(\alpha+\beta \gamma^{5}\right)= \\
& =I[-\tilde{A}(\alpha \bar{\alpha}+\bar{\beta} \beta)-C(\bar{\alpha} \beta+\bar{\beta} \alpha)]++\hat{p}[(1-B)(\alpha \bar{\alpha}-\beta \bar{\beta})-D(\bar{\alpha} \beta-\bar{\beta} \alpha)]+ \\
& +\gamma^{5}[-C(\bar{\alpha} \alpha+\bar{\beta} \beta)-\tilde{A}(\bar{\alpha} \beta+\bar{\beta} \alpha)]+\hat{p} \gamma^{5}[-D(\bar{\alpha} \alpha-\bar{\beta} \beta)+(1-B)(\bar{\alpha} \beta-\bar{\beta} \alpha)]
\end{aligned}
$$

allows to obtain the renormalized components of self-energy.

In our representation (4.6) it is sufficient to renormalize only one pole contribution, the second pole will obtain the correct properties automatically by substitution $W \rightarrow-W$. Looking at first term 
in spectral representation, we see that renormalization is divided into two parts: renormalization of eigenvalue and projector.

For stable fermion there is a physical requirement for projector. As it follows from (6.2) the projector at point $W=m$ has form

$$
\Pi_{1}^{\mathrm{r}}(m)=\frac{1}{2}\left[1-\gamma^{5} c+\frac{\hat{p}}{m}\left(b-\gamma^{5} d\right)\right]
$$

where parameters $b, d$ and $c$ are related by $b^{2}-d^{2}+c^{2}=1$. However, if $c \neq 0, d \neq 0$ then $\Pi_{1}^{\mathrm{r}}(m)$ do not commutate with spin projector, what leads to spin flip for fermion on mass shell. Therefore there are requirements for renormalization of a stable fermion:

$$
C^{\mathrm{r}}\left(m^{2}\right)=0, \quad D^{\mathrm{r}}\left(m^{2}\right)=0 .
$$

For unstable fermion, when pole is at point $W_{1}=M_{p}-\imath \Gamma_{p} / 2$, there is some arbitrariness. The simplest generalization of (6.4) consists in:

$$
C^{\mathrm{r}}\left(W_{1}^{2}\right)=0, \quad D^{\mathrm{r}}\left(W_{1}^{2}\right)=0 .
$$

The same relations arise from a principle, suggested in [10]: the chiral components should have poles with unit absolute value of residue.

A few words about the relation between renormalization constants $Z^{1 / 2}, \bar{Z}^{1 / 2}$. The pseudohermiticity condition

$$
\bar{Z}^{1 / 2}=\gamma^{0}\left(Z^{1 / 2}\right)^{\dagger} \gamma^{0}
$$

is traditionally used in literature, which is reduced to $\bar{\alpha}=\alpha^{*}, \bar{\beta}=-\beta^{*}$. However, as it was noted in [20], one should refused from this condition, if self-energy has absorptive parts. The same is seen from our renormalized propagator (6.3). Assuming pseudo-hermiticity we calculate $D^{\mathrm{r}}\left(W^{2}\right)$ thus:

$$
D^{\mathrm{r}}\left(W^{2}\right)=|\alpha|^{2}\left\{D\left(W^{2}\right)\left(1+\frac{|\beta|^{2}}{|\alpha|^{2}}\right)-\left(1-B\left(W^{2}\right)\right)\left(\frac{\beta}{\alpha}+\frac{\beta^{*}}{\alpha^{*}}\right)\right\} .
$$

Because $D\left(W^{2}\right)$ and $B\left(W^{2}\right)$ contain physically different contributions we cannot provide the condition $D^{\mathrm{r}}\left(W_{1}^{2}\right)=0$ for complex self-energy. So, the pseudo-hermiticity condition, tacitly assumed in [10], seems to be too restrictive for parity violating theory.

Let's consider below the case of CP conservative theory when component $C\left(p^{2}\right)=0$. In order to avoid CP violation under renormalization it is necessary to require (see (6.3))

$$
\bar{\alpha} \beta+\bar{\beta} \alpha=0 .
$$

The pseudo-hermiticity condition (6.6) leads to (6.8) in case of real $\alpha, \beta$ (stable fermion). However, for resonance one have to refuse from pseudo-hermiticity, (6.6).

Taking into account the condition (6.8) the renormalized inverse propagator becomes

$$
\begin{aligned}
S^{\mathrm{r}}=\alpha \bar{\alpha}\left\{-\tilde{A}\left(W^{2}\right)\left(1-x^{2}\right)+\hat{p}\left[\left(1-B\left(W^{2}\right)\right)\left(1+x^{2}\right)-D\left(W^{2}\right) 2 x\right]+\right. \\
\left.+\hat{p} \gamma^{5}\left[-D\left(W^{2}\right)\left(1+x^{2}\right)+\left(1-B\left(W^{2}\right)\right) 2 x\right]\right\},
\end{aligned}
$$


where $\alpha, \bar{\alpha}$ and $x=\beta / \alpha$ are complex numbers.

The condition at pole $D^{\mathrm{r}}\left(W_{1}^{2}\right)=0$ defines

$$
x \equiv \frac{\beta}{\alpha}=\frac{1-B_{1}-R_{1}}{D_{1}},
$$

where $B_{1}=B\left(W_{1}^{2}\right), D_{1}=D\left(W_{1}^{2}\right), R_{1}=R\left(W_{1}^{2}\right)$. Substituting that into $S^{\mathrm{r}}$, taking out common factor and denoting it by $Z$ we get

$$
\begin{aligned}
S^{\mathrm{r}}=Z\left\{-\tilde{A}\left(W^{2}\right)++\hat{p}[(1-\right. & \left.\left.B\left(W^{2}\right)\right) \frac{1-B_{1}}{R_{1}}-D\left(W^{2}\right) \frac{D_{1}}{R_{1}}\right]+ \\
& \left.+\hat{p} \gamma^{5}\left[-D\left(W^{2}\right) \frac{1-B_{1}}{R_{1}}+\left(1-B\left(W^{2}\right)\right) \frac{D_{1}}{R_{1}}\right]\right\}=\hat{p}-\Sigma^{\mathrm{r}},
\end{aligned}
$$

where renormalized components are given by

$$
\begin{aligned}
& \tilde{A}^{\mathrm{r}}\left(W^{2}\right)=Z \tilde{A}\left(W^{2}\right), \\
& B^{\mathrm{r}}\left(W^{2}\right)=1-Z\left[\left(1-B\left(W^{2}\right)\right) \frac{1-B_{1}}{R_{1}}-D\left(W^{2}\right) \frac{D_{1}}{R_{1}}\right], \\
& D^{\mathrm{r}}\left(W^{2}\right)=Z\left[D\left(W^{2}\right) \frac{1-B_{1}}{R_{1}}-\left(1-B\left(W^{2}\right)\right) \frac{D_{1}}{R_{1}}\right] .
\end{aligned}
$$

To determine $Z$ factor we consider renormalized eigenvalue $\lambda_{1}^{\mathrm{r}}(W)$, its derivative at $W=W_{1}$ has to equal 1 . It is easy to check that

$$
\lambda_{1}^{\mathrm{r}}(W)=Z \lambda_{1}(W)
$$

If to require $\left(\lambda_{1}^{\mathrm{r}}\right)^{\prime}\left(W_{1}\right)=1$ it gives

$$
Z=\frac{1}{R\left(W_{1}^{2}\right)+2 W_{1}^{2} R^{\prime}\left(W_{1}^{2}\right)-2 W_{1} A^{\prime}\left(W_{1}^{2}\right)} .
$$

In case of unstable fermions, the right hand side of (6.11) is, generally speaking, complex. If we define

$$
\lambda_{1,2}^{\mathrm{r}}(W)=|Z| \lambda_{1,2}(W),
$$

we have the renormalized propagator with $\lambda_{i}(W)$ satisfying the Schwartz principle,

$$
\lambda_{i}^{\mathrm{r}}\left(W^{*}\right)=\left(\lambda_{i}^{\mathrm{r}}(W)\right)^{*} .
$$

So, $\lambda_{i}^{\mathrm{r}}$ has zeroes at complex conjugate points $W_{1}, W_{1}^{*}$ with unit absolute value of residues.

\section{Conclusions}

We studied in detail the dressing of fermion propagator in the case of the parity non-conservation. In contrast to previous works, we found the representation of propagator (4.5), (5.7), where the positive and negative energy poles are separated from each other. We compared our resonance representation with Breit-Wigner form and used the on-shell definitions of mass and width. The spectral representation also allows to perform pole renormalization in a simple and compact way. 
We found that in case of parity violation the resonance factor (5.6) differs from Breit-Wignerlike formula. The reason is that in presence of $\gamma^{5}$ the Dyson summation of the self-energy insertions in a propagator takes another form. But in case of SM vertex the self-energy contains only the kinetic term and the obtained resonance factor $1 / \lambda_{1}(W)$ returns to the standard form for small width $\Gamma / m \ll 1$.

For top quark $\Gamma / m \sim 10^{-2}$ is really a small parameter, so for SM its resonance propagator will practically coincide with standard one. Recall that at LHC the measurement of $\Gamma_{\mathrm{t}}$ is a rather challenging problem, and it is difficult to observe the deviation from a standard picture in the form of a resonance curve.

Another possibility to see such a deviation is related with projectors (4.5). One sees that $\Pi_{k}$ do not commutate with spin projectors $\left(1 \pm \gamma^{5} \hat{s}\right) / 2$ and this fact can lead to non-trivial spin properties at the level of $\Gamma / m$.

We suppose that spectral representation (4.6) may be useful for neutrino propagation and mixing, if to consider it in QFT approach. If, following [21, 22], we are to consider neutrino propagation as macroscopic Feynman diagram, then at long distances only the positive energy contribution survives, and representation (4.6) allows one to identify covariantly this term.

\section{Acknowledgments}

We thank Slava Lee for participation at initial stage of the work and V.A. Naumov for discussion and valuable advice. The research is partially supported by the Federal Target Program "Research and Training Specialists in Innovative Russia, 2009-2013", Contract 14.B37.21.0910.

\section{References}

[1] W. Wagner, Top quark physics in hadron collisions, Rept. Prog. Phys. 68 (2005) 2409.

[2] A. Quadt, Top quark physics at hadron colliders, Eur. Phys. J. C48 (2006) 835.

[3] J. Incandela, A. Quadt, W. Wagner and Daniel Wicke, Status and Prospects of Top-Quark Physics, Prog. Part. Nucl. Phys. 63 (2009) 239.

[4] V. Khachatryan et al, First Measurement of the Cross Section for Top-Quark Pair Production in Proton-Proton Collisions at $\sqrt{s}=7 \mathrm{TeV}$, Phys. Lett. $\mathbf{B 6 9 5}$ (2011) 424.

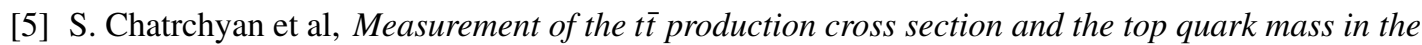
dilepton channel in pp collisions at $\sqrt{s}=7 \mathrm{TeV}$, JHEP 1107 (2011) 049.

[6] G. Aad et al, Measurement of the top quark-pair production cross section with ATLAS in pp collisions at $\sqrt{s}=7 \mathrm{TeV}$, Eur. Phys. J. C71 (2011) 1577.

[7] G. Aad et al, Measurement of the top quark pair production cross section in pp collisions at $\sqrt{s}=7$ $\mathrm{TeV}$ in dilepton final states with ATLAS, Phys. Lett. B707 (2012) 459.

[8] W. Bernreuther, Top quark physics at the LHC, J. Phys. G G35 (2008) 083001.

[9] A. Kaloshin and V. Lomov, The Rarita-Schwinger field: Dressing procedure and spin-parity content, Phys. Atom. Nucl. 69 (2006) 541.

[10] B. Kniehl and A. Sirlin, Pole Mass, Width, and Propagators of Unstable Fermions, Phys. Rev. D77 (2008) 116012. 
[11] A. Sirlin, Theoretical considerations concerning the Z0 mass, Phys. Rev. Lett. 67 (1991) 2127.

[12] A. Sirlin, Observations concerning mass renormalization in the electroweak theory, Phys. Lett. B267 (1991) 240 .

[13] M. Passera and A. Sirlin, Radiative corrections to $\mathrm{W}$ and quark propagators in the resonance region, Phys. Rev. D58 (1998) 113010.

[14] P. Gambino and P. Grassi, The Nielsen identities of the SM and the definition of mass, Phys. Rev. D62 (2000) 076002.

[15] M. Nekrasov, OMS-bar scheme of UV renormalization in the presence of unstable fundamental particles, Phys. Lett. B531 (2002) 225.

[16] A. Denner, Reduction of couplings in the two Higgs doublet extension of the electroweak standard model, Nucl. Phys. B347 (1990) 184.

[17] K. Aoki, Z. Hioki, M. Konuma, R. Kawabe and T. Muta, Electroweak Theory. Framework of On-Shell Renormalization and Study of Higher Order Effects, Prog. Theor. Phys. Suppl. 73 (1982) 1.

[18] A. Denner, Techniques for calculation of electroweak radiative corrections at the one loop level and results for W physics at LEP-200, Fortschr. Phys. 41 (1993) 307.

[19] D. Bardin and G. Passarino, The standard model in the making: Precision study of the electroweak interactions, International series of monographs on physics. 104 (1999).

[20] D. Espriu, J. Manzano and P. Talavera, Flavor mixing, gauge invariance and wave-function renormalisation, Phys. Rev. D66 (2002) 076002.

[21] W. Grimus and P. Stockinger, Effects of neutrino oscillations and neutrino magnetic moments on elastic neutrino-electron scattering, Phys. Rev. D57 (1998) 1762.

[22] W. Grimus, P. Stockinger and S. Mohanty, The Field theoretical approach to coherence in neutrino oscillations, Phys. Rev. D59 (1999) 013011. 